\title{
Distilling multipartite pure states from a finite number of copies of multipartite mixed states
}

\author{
Ping-Xing Chen ${ }^{1,2^{*}}$ and Cheng-Zu Li ${ }^{1}$ \\ ${ }^{1}$ Department of Applied Physics, National University of Defense \\ Technology, Changsha, 410073, P. R. China. \\ ${ }^{2}$ Key Laboratory of Quantum Information, University of Science and \\ Technology of China, Chinese Academy of Sciences, Hefei 230026, P. R. China
}

\begin{abstract}
This paper will address the question of the distillation of entanglement from a finite number of multi-partite mixed states. It is shown that if one can distill a pure entangled state from $n$ copies of a mixed state $\sigma_{A B C} \ldots$ there must be at least a subspace in whole Hilbert space of the all copies such that the projection of $\sigma_{A B C \ldots}^{\otimes n}$ onto the subspace is a pure entangled state. We also show that the purification of entanglement or distillation of entanglement can be carried out by local joint projective measurements with the help of classical communication and local general positive operator valued measurements on a single particle, in principle. Finally we discuss experimental realizability of the entanglement purification.
\end{abstract}

PACS: 03.67.-a, 03.65.Bz.

A pure entangled state plays perhaps a central role in quantum information. It not only gives rise to some completely new applications, such as error correcting code [1], dense coding [2] and teleportation [3], but also has been a source of great theoretical interest, such as nonlocality [4] and the experimental tests of Bell theorem [5]. However, interactions with the environment always occur, and a pure entangled state will became into a mixed state which will degrade the quality of the entanglement. This raises a problem: how to distill a pure entangled state from mixed states? Bennett et al [6,7] first proposed an entanglement purification scheme for a class of two-qubits mixed states. This scheme needs infinite copies of the mixed state, in principle, and needs collective measurements [8]. Horodecki et al [9] proved that all mixed entangled states of two qubits can be purified into a singlet if infinite copies of the mixed state are provided. However, in practice one cannot get infinite copies of a mixed states. Can one get a pure entangled state from finite copies of a mixed state? Linden et al [8] showed that one cannot get a pure entangled state from an individual Werner state by local operations and classical communication (LOCC). Hent [10] generalized this result and showed that no scheme can produce a maximally entangled state of a bipartite system from a generic mixed state of the system. Recently, we considered the condition for

*E-mail: pxchen@nudt.edu.cn distillability from finite copies of a mixed state of arbitrary bipartite system [11], and showed that one can get a pure entangled state from $\mathrm{n}$ copies of a mixed state if and only if there exists at least a subspace the projection of $\mathrm{n}$ copies onto this subspace is a pure entangled state. We defined the subspace as distillable subspace (DSS). DSS is similar to the decoherence-free space [12].

This paper will consider the distillation of the entanglement from a single or finite copies of a multi-partite mixed state. We first analysis the property of operators for the distillation of entanglement or the purification of entanglement, and show that any local operation can be regarded as a combination of three classes of operators, i.e., local projective operators (LPO), local filter operators (LFO) and local unitary operators (LUO). Then we prove that if one can distill a pure entangled state from $\mathrm{n}$ copies of a mixed state $\sigma_{A B C \ldots}$ there must be at least a subspace of whole Hilbert space of the all copies such that the projection of $\sigma_{A B C \ldots}^{\otimes n}$ onto the subspace is a pure entangled state. We also show that any operation for the entanglement purification or the entanglement distillation acting on a Hilbert space of a quantum system is, in essence, to first project out a subspace which has a pure entangled state or a highly entangled mixed states, then change the entanglement of the state projected out. It is shown that only LPO may produce a pure entangled state from a or many mixed states; LFO may increase the entanglement of a entangled state, but never can transfer a mixed states into a pure state. Finally we discuss the experimental realizability of the entanglement purification.

Consider a mixed entangled state $\sigma_{A B C \ldots}$ shared by separated parties Alice, Bob and Charles et al. Now we hope distill a pure entangled state $|\Psi\rangle$ from $n$ copies of state $\sigma_{A B C} \ldots$. Any protocol for the distillation of entanglement from $n$ copies of a mixed state $\sigma_{A B C \ldots}, \sigma_{A B C \ldots}^{\otimes n}$, can be conceived as successive rounds of measurements and communication by Alice, Bob and Charles et al. After rounds of measurements and communication, there are many possible outcomes which correspond to many measurement operators $\left\{A_{i} \otimes B_{i} \otimes C_{i} \otimes \cdots\right\}$ acting on the Alice, Bob and Charles's et al Hilbert space. Each of these operators is a product of the positive operators and unitary maps corresponding to Alice's, Bob's and Charles's measurements and rotations, and represents the effect of the $\mathrm{N}$ measurements and communication. 
If the outcome $i$ occurs, the given state $\sigma_{A B C \ldots}^{\otimes n}$ becomes:

$$
A_{i} \otimes B_{i} \otimes C_{i} \otimes \cdots \sigma_{A B C \cdots}^{\otimes n} A_{i}^{+} \otimes B_{i}^{+} \otimes C_{i}^{+} \otimes \cdots
$$

If one can distill a pure entangled state from $\sigma_{A B C \ldots}^{\otimes n}$, there must be at least an element $A \otimes B \otimes C \otimes \cdots \in$ $\left\{A_{i} \otimes B_{i} \otimes C_{i} \otimes \cdots\right\}$ such that

$A \otimes B \otimes C \otimes \cdots \sigma_{A B C \ldots}^{\otimes n} A^{+} \otimes B^{+} \otimes C^{+} \otimes \cdots \rightarrow|\Psi\rangle\langle\Psi|$,

where $|\Psi\rangle$ is a pure entangled state. Operator $A, B, C$ can be always expressed as:

$$
\begin{aligned}
& A=a_{1}\left|\phi_{1}^{\prime}\right\rangle\left\langle\phi_{1}\left|+\cdots+a_{n_{a}}\right| \phi_{n_{a}}^{\prime}\right\rangle\left\langle\phi_{n_{a}}\right| ; \\
& B=b_{1}\left|\xi_{1}^{\prime}\right\rangle\left\langle\xi_{1}\left|+\cdots+b_{n_{b}}\right| \xi_{n_{b}}^{\prime}\right\rangle\left\langle\xi_{n_{b}}\right| ; \\
& C=c_{1}\left|\eta_{1}^{\prime}\right\rangle\left\langle\eta_{1}\left|+\cdots+c_{n_{c}}\right| \eta_{n_{c}}^{\prime}\right\rangle\left\langle\eta_{n_{c}}\right| ;
\end{aligned}
$$

where $\left\{\left|\phi_{j}^{\prime}\right\rangle, j=1, \cdots, n_{a}\right\},\left\{\left|\phi_{j}\right\rangle, j=1, \cdots, n_{a}\right\}$ are Alice's two sets of orthogonal vectors; $\left\{\left|\xi_{l}^{\prime}\right\rangle, l=1, \cdots, n_{b}\right\},\left\{\left|\xi_{l}\right\rangle, l=1, \cdots, n_{b}\right\}$ are Bob's two sets of orthogonal vectors; $\left\{\left|\eta_{p}^{\prime}\right\rangle, p=1, \cdots, n_{c}\right\},\left\{\left|\eta_{p}\right\rangle, p=1, \cdots, n_{c}\right\}$ are Charles's two sets of orthogonal vectors. $0<a_{j} \leq 1$, $j=1, \cdots, n_{a} ; 0<b_{l} \leq 1, l=1, \cdots, n_{b} ; 0<c_{p} \leq 1, p=$ $1, \cdots, n_{c}$.

The operator $A$ in (3) can be carried out by following three operators: 1) a LPO $P_{A}=\left|\phi_{1}\right\rangle\left\langle\phi_{1}\right|+\cdots+$ $\left|\phi_{n_{a}}\right\rangle\left\langle\phi_{n_{a}}\right|$ which projects out a subspace spanned by bases $\left.\left|\phi_{j}\right\rangle, j=1, \cdots, n_{a} ; 2\right)$ a LFO $F_{A}=a_{1}\left|\phi_{1}\right\rangle\left\langle\phi_{1}\right|+$ $\cdots+a_{n_{a}}\left|\phi_{n_{a}}\right\rangle\left\langle\phi_{n_{a}}\right|$ which changes the relative weights of the components $\left.\left|\phi_{j}\right\rangle, j=1, \cdots, n_{a} ; 3\right)$ a LUO which transfers the Alice's vectors from $\left\{\left|\phi_{j}\right\rangle, j=1, \cdots, n_{a}\right\}$ to $\left\{\left|\phi_{j}^{\prime}\right\rangle, j=1, \cdots, n_{a}\right\}$, and similarly for $B$ and $C$. This is the character of the operators for the distillation of entanglement we shall use below.

Definitions: If a pure state $|\Psi\rangle_{A B C \ldots}$ has reduced density matrices $\sigma_{A}, \sigma_{B}, \sigma_{C}, \cdots$ with ranks $n_{A}, n_{B}, n_{C}, \cdots$, respectively, we say $|\Psi\rangle_{A B C \ldots}$ is an $n_{A} \otimes$ $n_{B} \otimes n_{C} \otimes \cdots$ state, where $\sigma_{A}=\operatorname{tr}_{B C \ldots}\left(|\Psi\rangle_{A B C \ldots}\langle\Psi|\right)$, and similarly for $\sigma_{B}, \sigma_{C}, \cdots$. If $|\Psi\rangle_{A B C \ldots}$ and $|\Phi\rangle_{A B C \ldots}$ are of $n_{A} \otimes n_{B} \otimes n_{C} \otimes \cdots$ states, we say $|\Psi\rangle_{A B C \ldots}$ is same dimension as $|\Phi\rangle_{A B C \ldots}$

Lemma 1: If two mixed states $\rho_{A B C \ldots}$ and $\rho_{A B C \ldots}^{\prime}$ in a Hilbert space $H=H_{A} \otimes H_{B} \otimes H_{C} \otimes \cdots$ fulfill

$$
A \otimes B \otimes C \otimes \cdots \rho_{A B C} \ldots A^{+} \otimes B^{+} \otimes C^{+} \otimes \cdots=\rho_{A B C \ldots}^{\prime},
$$

where $A, B, C$ are full rank operators to Hilbert spaces $H_{A}, H_{B}, H_{C}, \cdots$, respectively, then the rank of $\rho_{A B C \cdots}$ is equal to that of $\rho_{A B C \ldots}^{\prime}$, i.e., $r\left(\rho_{A B C \ldots}\right)=r\left(\rho_{A B C \ldots}^{\prime} \ldots\right)$.

Proof: We consider a set of eigenstates-decomposition of state $\rho_{A B C \cdots}$

$$
\begin{aligned}
\rho_{A B C \ldots} & =\sum_{i=1}^{r\left(\rho_{A B C \ldots}\right)} p_{i}\left|\Psi^{i}\right\rangle_{A B C \ldots}\left\langle\Psi^{i}\right|, \\
p_{i} & >0 ; \sum_{i} p_{i}=1 .
\end{aligned}
$$

Note that

$$
A \otimes B \otimes C \otimes \cdots\left|\Psi^{i}\right\rangle_{A B C \ldots}=\left|\Psi^{i}\right\rangle_{A B C \ldots},
$$

then

$$
\rho_{A B C \ldots}^{\prime}=\sum_{i=1}^{r\left(\rho_{A B C \ldots} \ldots\right)} p_{i}\left|\Psi^{\prime i}\right\rangle_{A B C \ldots}\left\langle\Psi^{\prime i}\right| .
$$

Obviously, we have that

$$
r\left(\rho_{A B C \ldots}\right) \geqslant r\left(\rho_{A B C \ldots}^{\prime} \ldots\right) .
$$

We introduce operators $A^{\prime}, B^{\prime}, C^{\prime}$

$$
A^{\prime}=a_{1}^{\prime}\left|\phi_{1}\right\rangle\left\langle\phi_{1}^{\prime}\left|+\cdots+a_{n_{a}}^{\prime}\right| \phi_{n_{a}}\right\rangle\left\langle\phi_{n_{a}}^{\prime}\right|
$$

such that $a_{1}^{\prime} a_{1}=\cdots=a_{n_{a}}^{\prime} a_{n_{a}}$, and similarly for $B^{\prime}, C^{\prime}$. Since operators $A, B, C$ and $A^{\prime}, B^{\prime}, C^{\prime}$ are full rank, we have that

$$
A^{\prime} \otimes B^{\prime} \otimes C^{\prime} \otimes \cdots\left|\Psi^{\prime i}\right\rangle_{A B C \ldots} \propto\left|\Psi^{i}\right\rangle_{A B C \ldots} .
$$

Thus there exists an operator $A^{\prime} \otimes B^{\prime} \otimes C^{\prime} \otimes \cdots$ such that

$$
\begin{aligned}
& A^{\prime} \otimes B^{\prime} \otimes C^{\prime} \otimes \cdots \rho_{A B C \ldots}^{\prime} A^{\prime+} \otimes B^{\prime+} \otimes C^{\prime+} \otimes \cdots \\
= & \sum_{i=1}^{r\left(\rho_{A B C} \ldots\right)} p_{i}^{\prime}\left|\Psi^{i}\right\rangle_{A B C \ldots}\left\langle\Psi^{i}\right|, \quad p_{i}^{\prime}>0 ; \quad \sum_{i} p_{i}^{\prime}=1,
\end{aligned}
$$

this follows that

$$
r\left(\rho_{A B C \ldots}\right) \leq r\left(\rho_{A B C \ldots}^{\prime}\right) .
$$

Combinning (8) and (12) we finish the proof of Lemma 1.

If one hope distill a pure state with Schmidt numbers n from a bipartite mixed state $\sigma \in C^{n} \otimes C^{n}$, the operator for this distillation should be full rank, obviously. From Lemma 1 we can follow that this kind of distillation is impossible as shown in Ref [10]

Lemma 2: If two pure states $|\Psi\rangle_{A B C \ldots}$ and $|\Phi\rangle_{A B C \ldots}$ in a Hilbert space $H=H_{A} \otimes H_{B} \otimes H_{C} \otimes \cdots$ fulfill

$$
A \otimes B \otimes C \otimes \cdots|\Psi\rangle_{A B C \ldots}=|\Phi\rangle_{A B C \ldots},
$$

where $A, B, C$ are full rank operators to Hilbert spaces $H_{A}, H_{B}, H_{C}, \cdots$, respectively, then the ranks of the corresponding reduced density matrices satisfy $r\left(\rho_{A}^{\Psi}\right)=$ $r\left(\rho_{A}^{\Phi}\right), r\left(\rho_{B}^{\Psi}\right)=r\left(\rho_{B}^{\Phi}\right)$ and $r\left(\rho_{C}^{\Psi}\right)=r\left(\rho_{C}^{\Phi}\right)$.

Proof: As shown in appendix A in Ref. [13], if (13) holds, then $r\left(\rho_{i}^{\Psi}\right) \geqslant r\left(\rho_{i}^{\Phi}\right), i=A, B, C$. By the similar proof as that of Lemma 1, we can introduce operators 
$A^{\prime}, B^{\prime}, C^{\prime}$ in (9). Since operators $A, B, C$ and $A^{\prime}, B^{\prime}, C^{\prime}$ are full rank, we have that

$$
A^{\prime} \otimes B^{\prime} \otimes C^{\prime} \otimes \cdots|\Phi\rangle_{A B C \ldots} \propto|\Psi\rangle_{A B C \ldots} .
$$

Thus $r\left(\rho_{i}^{\Psi}\right) \leq r\left(\rho_{i}^{\Phi}\right), i=A, B, C$, and then $r\left(\rho_{i}^{\Psi}\right)=$ $r\left(\rho_{i}^{\Phi}\right), i=A, B, C$. This ends the proof.

Theorem 1: Alice, Bob and Charles et al can distill an $n_{A} \otimes n_{B} \otimes n_{C} \otimes \cdots$ pure state $|\Psi\rangle_{A B C \ldots}$ from $n$ copies of a mixed state $\sigma_{A B C} \ldots$ if and only if there is at least one subspace $H_{A}^{\prime} \otimes H_{B}^{\prime} \otimes H_{C}^{\prime} \otimes \cdots, H_{A}^{\prime} \in H_{A}^{\otimes n}$, $H_{B}^{\prime} \in H_{B}^{\otimes n}, H_{C}^{\prime} \in H_{C}^{\otimes n}$, such that the projection of state $\sigma_{A B C \ldots}^{\otimes n}$ on this subspace is an $n_{A} \otimes n_{B} \otimes n_{C} \otimes \cdots$ pure state $\left|\Psi^{\prime}\right\rangle_{A B C \ldots}$ or $|\Psi\rangle_{A B C \ldots}$, and $\left|\Psi^{\prime}\right\rangle_{A B C \ldots}$ can be transferred into the state $|\Psi\rangle_{A B C \ldots}$ by LFO and LUO with nonzero probability.

Proof: If there is a subspace $H_{A}^{\prime} \otimes H_{B}^{\prime} \otimes H_{C}^{\prime} \otimes \cdots$ satisfying the condition in theorem 1 above, Alice, Bob and Charles can use projective operators $P_{A}, P_{B}$ and $P_{C}$ which project out subspace $H_{A}^{\prime}, H_{B}^{\prime}, H_{C}^{\prime}$, respectively, to get an $n_{A} \otimes n_{B} \otimes n_{C} \otimes \cdots$ pure state $|\Psi\rangle_{A B C \ldots}$ or $\left|\Psi^{\prime}\right\rangle_{A B C \ldots}$. If they get the state $\left|\Psi^{\prime}\right\rangle_{A B C \ldots}$, they can transferred the state $\left|\Psi^{\prime}\right\rangle_{A B C \ldots}$ into the state $|\Psi\rangle_{A B C \ldots}$ with nonzero probability by LFO and LUO. Let's then move to prove the converse. If Alice, Bob and Charles et al can distill an $n_{A} \otimes n_{B} \otimes n_{C} \otimes \cdots$ pure state $|\Psi\rangle_{A B C \cdots}$ from $n$ copies of a mixed state $\sigma_{A B C} \ldots$, there must be an operator $A \otimes B \otimes C \otimes \cdots$ such that

$$
\begin{aligned}
& A \otimes B \otimes C \otimes \cdots \sigma_{A B C \cdots}^{\otimes n} A^{+} \otimes B^{+} \otimes C^{+} \otimes \cdots \\
\rightarrow & |\Psi\rangle_{A B C \cdots}\langle\Psi| .
\end{aligned}
$$

Note that projective operators $P_{A}, P_{B}, P_{C}, \cdots$

$$
\begin{gathered}
P_{A}=\left|\phi_{1}\right\rangle\left\langle\phi_{1}|+\cdots+| \phi_{n_{a}}\right\rangle\left\langle\phi_{n_{a}}\right| ; \\
P_{B}=\left|\xi_{1}\right\rangle\left\langle\xi_{1}|+\cdots+| \xi_{n_{b}}\right\rangle\left\langle\xi_{n_{b}}\right| ; \\
P_{C}=\left|\eta_{1}\right\rangle\left\langle\eta_{1}|+\cdots+| \eta_{n_{c}}\right\rangle\left\langle\eta_{n_{c}}\right|,
\end{gathered}
$$

act in the subspaces $H_{A}^{\prime}, H_{B}^{\prime}$ and $H_{C}^{\prime}$ spanned by bases $\left\{\left|\phi_{1}\right\rangle, \cdots,\left|\phi_{n_{a}}\right\rangle\right\}_{A},\left\{\left|\xi_{1}\right\rangle, \cdots,\left|\xi_{n_{b}}\right\rangle\right\}_{B} \quad$ and $\left\{\left|\eta_{1}\right\rangle, \cdots,\left|\eta_{n_{c}}\right\rangle\right\}_{C}$, respectively. Then, (15) can became

$$
\begin{aligned}
& A \otimes B \otimes C \otimes \cdots\left(P_{A} \otimes P_{B} \otimes P_{C} \otimes \cdots\right. \\
& \left.\sigma_{A B C \ldots}^{\otimes n} P_{A} \otimes P_{B} \otimes P_{C} \otimes \cdots\right) A^{+} \otimes B^{+} \otimes C^{+} \otimes \cdots \\
\rightarrow & |\Psi\rangle_{A B C \ldots}\langle\Psi| .
\end{aligned}
$$

$P_{A} \otimes P_{B} \otimes P_{C} \otimes \cdots \sigma_{A B C \ldots}^{\otimes n} P_{A} \otimes P_{B} \otimes P_{C} \otimes \cdots$ noted as $\sigma_{A B C \ldots}^{\prime \otimes n}$ is a projection of state $\sigma_{A B C \ldots}^{\otimes n}$ onto the subspace $H_{A}^{\prime} \otimes H_{B}^{\prime} \otimes H_{C}^{\prime}$. The effect of operator $A \otimes B \otimes C \otimes \cdots$ is just to do local unitary operators and local filter operators on whole subspace $H_{A}^{\prime} \otimes H_{B}^{\prime} \otimes H_{C}^{\prime}$. Since operators $P_{A}, P_{B}, P_{C}$ are full-rank to subspaces $H_{A}^{\prime}, H_{B}^{\prime}, H_{C}^{\prime}$, respectively, because of Lemma $1 P_{A} \otimes P_{B} \otimes P_{C}$ cannot convert a mixed state in subspace $H_{A}^{\prime} \otimes H_{B}^{\prime} \otimes H_{C}^{\prime}$ into a pure state. So state $\sigma_{A B C \ldots}^{\otimes n}$ is a pure state. Because of Lemma 2 and $A, B, C$ being full rank to subspace $H_{A}^{\prime} \otimes H_{B}^{\prime} \otimes H_{C}^{\prime}, \sigma_{A B C \ldots}^{\prime \otimes n}$ is an $n_{A} \otimes n_{B} \otimes n_{C} \otimes \cdots$ pure state. From (15) we can follow that $\sigma_{A B C \ldots}^{\prime \otimes n}$ is the state $|\Psi\rangle_{A B C \ldots}\langle\Psi|$ or can be converted into $|\Psi\rangle_{A B C \ldots}\langle\Psi|$ by LFO and LUO. This ends the proof.

Note that for two pure states $|\Psi\rangle$ and $|\Phi\rangle$ in bipartite system, if they have same Schmidt numbers they can be transferred into each other by local filter operators. But for multi-partite system, two $n_{A} \otimes n_{B} \otimes n_{C} \otimes \cdots$ pure states may belong to two inequivalent classes, such as three qubits's GHZ state [14] and W state [13].

$$
\begin{array}{r}
|G H Z\rangle=\frac{1}{\sqrt{2}}(|000\rangle+|111\rangle) ; \\
|W\rangle=\frac{1}{\sqrt{3}}(|100\rangle+|010\rangle+|011\rangle)
\end{array}
$$

Theorem 1 above shows that if one can distill a pure entangled state from a single mixed state $\sigma_{A B C} \ldots$ there must be at least a subspace such that the projection of the state $\sigma_{A B C \ldots}$ onto the subspace is a pure entangled state, i.e., there is at least a DSS. To get a desired pure entangled state, one can first get a pure entangled state by LPO, then change the pure entangled state into a desired state by LFO and LUO acting on the subspace projected out by LPO.

Theorem 2: If one can get an $n_{A} \otimes n_{B} \otimes n_{C} \otimes$ $\cdots$ pure entangled state from the $n$ copies of a mixed state $\sigma_{A B C \ldots}$, the rank of $\sigma_{A B C \ldots}^{\otimes n}$ is at most $\left(\operatorname{dim} H_{A} \cdot \operatorname{dim} H_{B} \cdot \operatorname{dim} H_{C}\right)^{n}-n_{A} \cdot n_{B} \cdot n_{C} \cdots+1$.

Proof: If one can get an $n_{A} \otimes n_{B} \otimes n_{C} \otimes \cdots$ pure entangled state from $n$ copies of a mixed state $\sigma_{A B C} \ldots$ there is at least a DSS $H=H_{A}^{\prime} \otimes H_{B}^{\prime} \otimes H_{C}^{\prime}, H_{i}^{\prime} \in H_{i}^{\otimes n}, i=$ $A, B, C$, and obviously $\operatorname{dim} H_{i}^{\prime} \geqslant n_{i}, i=A, B, C$. We always can write down the matrix $\left[\sigma_{A B C \ldots}^{\otimes n}\right]$ under the bases including the full orthogonal bases in the subspace $H^{\perp}$ orthogonal to the DSS $H$, and the orthogonal bases in the DSS $H$. Since the projection of $\sigma_{A B C \ldots}^{\otimes n}$ onto the DSS $H$ is an $n_{A} \otimes n_{B} \otimes n_{C} \otimes \cdots$ pure state $|\Psi\rangle$, if we choose a set of orthogonal vectors including $|\Psi\rangle$ as the bases of the DSS $H$, then the matrix $\left[\sigma_{A B C \ldots}^{\otimes n}\right]$ is at least $\operatorname{dim} H_{A}^{\prime}$. $\operatorname{dim} H_{B}^{\prime}$. $\operatorname{dim} H_{C}^{\prime}-1$ rows zero elements and $\operatorname{dim} H_{A}^{\prime}$. $\operatorname{dim} H_{B}^{\prime}$. $\operatorname{dim} H_{C}^{\prime}-1$ columns zero elements. Thus the rank of $\sigma_{A B C \ldots}^{\otimes n}$ is at most

$$
\begin{aligned}
& \left(\operatorname{dim} H_{A} \cdot \operatorname{dim} H_{B} \cdot \operatorname{dim} H_{C}\right)^{n}- \\
& \operatorname{dim} H_{A}^{\prime} \cdot \operatorname{dim} H_{B}^{\prime} \cdot \operatorname{dim} H_{C}^{\prime}+1 \\
\leq & \left(\operatorname{dim} H_{A} \cdot \operatorname{dim} H_{B} \cdot \operatorname{dim} H_{C}\right)^{n}-n_{A} \cdot n_{B} \cdot n_{C} \cdots+1 .
\end{aligned}
$$

This ends the proof.

Let's now consider the role of LPO and LFO in the entanglement purification or the entanglement distillation, respectively. As shown before, any operator $A \otimes B \otimes C \otimes \cdots$ describing local POV measures can be 
regarded as a combination of three classes of operators (namely, LPO, LFO and LUO). In the case of entanglement distillation if an operator $A \otimes B \otimes C \otimes \cdots$ can bring about a pure entangled state $|\Psi\rangle$ from n copies of a mixed state, it is the LPO that bring about a pure entangled state as same dimension as $|\Psi\rangle$. Because of the Lemma 1, the local filter operator acting on the whole subspace projected out by LPO can change the entanglement of the pure state but not can transfer a mixed state into a pure state. However, if we turn to the problem of whether the entanglement of a mixed state $\sigma_{A B C} \ldots$ can be increased by LOCC, we find only local filter operator may increase the entanglement of $\sigma_{A B C} \ldots$ with non-zero probability. The proof is simple. Let's take the formation of entanglement as an example. A mixed state $\sigma_{A B C \ldots}$ always can be expressed as follows [15]:

$$
\sigma_{A B C \ldots}=\sum_{i} p_{i}\left|\Phi_{i}\right\rangle\left\langle\Phi_{i}\right|
$$

where $\left|\Phi_{i}\right\rangle s$ is a set of pure states decomposition of $\sigma_{A B C \ldots}$ such that $\sum_{i} p_{i} E\left(\left|\Phi_{i}\right\rangle\right)$ is a minimum over all possible decompositions, and thus the formation of entanglement $E_{F}\left(\sigma_{A B C \ldots}\right)$ of $\sigma_{A B C \ldots}$ is $\sum_{i} p E\left(\left|\Phi_{i}\right\rangle\right)$. $E\left(\left|\Phi_{i}\right\rangle\right)$ is the entanglement of the pure state $\left|\Phi_{i}\right\rangle$ ( we now do not know the entanglement of a multi-partite pure state, but here we can note the entanglement of $\left|\Phi_{i}\right\rangle$ is $\left.E\left(\left|\Phi_{i}\right\rangle\right)\right)$. Any projective operator cannot increase the entanglement of the pure state $\left|\Phi_{i}\right\rangle$, and cannot increase the $E_{F}\left(\sigma_{A B C \ldots} \ldots\right)$ of $\sigma_{A B C} \ldots$. It is the LFO that may increase the entanglement of a mixed state. For example, a mixed state, $\sigma=\lambda\left[\frac{\sqrt{3}}{2}|00\rangle+\frac{1}{2}|11\rangle\right]+(1-\lambda)[|01\rangle]$, can be transferred into a mixed state $\sigma^{\prime}=\lambda^{\prime}\left[\frac{\sqrt{2}}{2}(|00\rangle+|11\rangle)\right]+$ $\left(1-\lambda^{\prime}\right)[|01\rangle]$ by operator $A=\frac{1}{2}|0\rangle_{A}\left\langle 0\left|+\frac{\sqrt{3}}{2}\right| 1\right\rangle_{A}\langle 1|$. Employing the Wootters's formula [15] for calculating the formation of entanglement, we can find that when $0.9 \leq \lambda<1, E_{F}\left(\sigma^{\prime}\right)>E_{F}(\sigma)$. Of course, as claimed in Ref. $[8,10]$ the entanglement of some mixed states, such as Werner states, cannot be increased by local filter operator.

Let's now look at the role of collective measurements in the entanglement distillation or the entanglement purification. It is possible that a mixed state $\sigma$ has no DSS, but $\sigma^{\otimes n}$ has DSS or $\sigma^{\otimes n}$ has a subspace the projection of $\sigma^{\otimes n}$ onto the subspace is a mixed entangled state with more entanglement than $\sigma$. An example of entanglement distillation is a three qubits' mixed state

$$
\begin{aligned}
\sigma= & p\left[\frac{1}{\sqrt{2}}\left(|0\rangle_{A}|0\rangle_{B}|0\rangle_{C}+|1\rangle_{A}|1\rangle_{B}|1\rangle_{C}\right)\right]+ \\
& (1-p)\left[|0\rangle_{A}|1\rangle_{B}|1\rangle_{C}\right]
\end{aligned}
$$

which has no DSS. But $\sigma^{\otimes 2}$ has a DSS the projection of $\sigma^{\otimes 2}$ onto this DSS is a pure state $|\Phi\rangle=$ $\frac{1}{\sqrt{2}}\left(|01\rangle_{A}|01\rangle_{B}|01\rangle_{C}+|10\rangle_{A}|10\rangle_{B}|10\rangle_{C}\right)$ which can be distilled by projective operators $P_{i}=|01\rangle_{i}\langle 01|+$ $|10\rangle_{i}\langle 10|, i=A, B, C$. The pure state $|\Phi\rangle$ is shared by six qubits. To make the state $|\Phi\rangle$ be shared by three qubits, we can first use unitary rotation operations on one of two qubits of A, B, C, respectively. i.e., $|0\rangle_{i} \rightarrow$ $\frac{1}{\sqrt{2}}\left(\left|0^{\prime}\right\rangle_{i}+\left|1^{\prime}\right\rangle_{i}\right),|1\rangle_{i} \rightarrow \frac{1}{\sqrt{2}}\left(\left|0^{\prime}\right\rangle_{i}-\left|1^{\prime}\right\rangle_{i}\right), i=A, B, C$, then measure the rotated qubits by local projective measurements. Thus the remained three qubits of A, B, C share a $|G H Z\rangle$ state by using a local phase flip operation. An example of entanglement purification is a Werner state $\sigma$,

$$
\sigma=F\left[\left|\Phi^{+}\right\rangle\right]+\frac{1-F}{3}\left(\left[\left|\Phi^{-}\right\rangle\right]+\left[\left|\Psi^{+}\right\rangle\right]+\left[\left|\Psi^{-}\right\rangle\right]\right)
$$

where $\left|\Phi^{ \pm}\right\rangle=\frac{1}{\frac{1}{\sqrt{2}}\left(|0\rangle_{A}|0\rangle_{B}\right.} \quad \pm$
$\left.|1\rangle_{A}|1\rangle_{B}\right) ;\left|\Psi^{ \pm}\right\rangle=\frac{1}{\sqrt{2}}\left(|0\rangle_{A}|1\rangle_{B} \pm|1\rangle_{A}|0\rangle_{B}\right) . \sigma^{\otimes 2}$ has two subspaces, one spanned by Alice's bases $|01\rangle_{A},|10\rangle_{A}$ and Bob's bases $|01\rangle_{B},|10\rangle_{B}$, another spanned by Alice's bases $|00\rangle_{A},|11\rangle_{A}$ and Bob's bases $|00\rangle_{B},|11\rangle_{B}$. The projection of $\sigma^{\otimes 2}$ onto the two subspaces are a Bell-diagonal state $\sigma^{\prime}$ with more entanglement than $\sigma$. The two examples above means that in the collection measurements the role of $n$ copies is, in essence, to provide a bigger Hilbert space where there may exist a DSS or a desired subspace.

Let's now discuss experimental realizability of the entanglement purification or the entanglement distillation. The scheme in previous papers $[6,7]$ needs very delicate C-NOT operators among different particles. This is disappointing from an experimental point of view [10]. Our results show this is not always true. If we can project out the DSS with a joint projective measurement on particles of two pairs, then the C-NOT is not necessary, in principle. For example, a new scheme of purification proposed recently by pan et al [16] used a efficient joint projective measurements. However, we still cannot say our results is inspiring from the experimental point of view for the following reasons: 1 . the theorem 1 shows that distilling a pure entangled state requires that a projection of the original state is pure, namely requires the original state has DSS. This is a very strong condition which can be satisfied only in the cases where some especial noise is present [11]; 2. while the multiple copies of a state which has no DSS may have DSS or desired subspaces, realization of the multiple copies is very hard although it has been demonstrated for some systems [16].

In summary, we discuss the condition for entanglement distillation from finite copies of multi-partite mixed state, and show that one can distill a pure entangled state from $\mathrm{n}$ copies of a mixed state $\sigma_{A B C} \ldots$ if and only if there must be at least a subspace such that the projection of the state $\sigma_{A B C \ldots}^{\otimes n}$ onto the subspace is a pure entangled state. This is a very strong condition and cannot be satisfied in many cases. If this condition is satisfied and the multiple copies of a state can be realized the purification or distillation of entanglement can be carried out by joint projective measurements and local POVM on a single particle with 
the help of classical communication, in principle.

[1] C. H. Bennett, D. P. Divincenzo, J. A.Smolin, and W. K.Wootters, Phys. Rev. A 54, 3824 (1996).

[2] C.H. Bennett and S.J. Wiesner, Phys.Rev.Lett.69,2881 (1992).

[3] C.H. Bennett, G.Brassard, C.Crepeau, R.Jozsa, A.Peres and W.K.Wootters, Phys.Rev.Lett.70,1895 (1993).

[4] Barbara M. Terhal, Andrew C. Doherty, David Schwab, Phys. Rev. Lett 90, 157903 (2003)

[5] Homas Jennewein, Gregor Weihs, Jian-Wei Pan, Anton Zeilinger, Phys. Rev. Lett. 88, 017903 (2002)

[6] C. H. Bennett, G. Brassard, S. Popescu, B. Schumacher and W. K. Wootters, Phys. Rev. Lett 76722 (1996)

[7] C. H. Bennett, G. Brassard, S. Popescu and B. Schumacher, Phys. Rev. A 532046 (1996).

[8] N. Linden, S. Massar and S. Popescu, Phys. Rev. Lett 81, 3279 (1998)

[9] M. Horodecki, P. Horodecki, and R. Horodecki, Phys. Rev. Lett 78, 574 (1997).

[10] Adrian Kent, Phys.Rev.Lett. 81, 2839-2841 (1998)

[11] P.-X.Chen, L.-M Liang, C.-Z Li and M.Q Huang, Phys.Rev.A65, 012317(2002); Phys.Rev.A66, 022309(2002)

[12] Lu-Ming Duan, Guang-Can Guo Phys. Rev. Lett. 79, 1953 (1997)

[13] W. Dur, G. Vidal, J. I. Cirac, Phys. Rev. A 62, 062314 (2000)

[14] D. M. Greenberger, M. Horne, A. Zeilinger, In Bell's theorem, Quantum Theory and Conception of the univers, edited by M. Kafatos et al (1989)

[15] William K. Wootters, Phys.Rev.Lett. 80, 2245-2248 (1998)

[16] Jian-Wei Pan, Christoph Simon, Caslav Brukner, Anton Zeilinger, Nature 410, 1067-1070 (2001) 